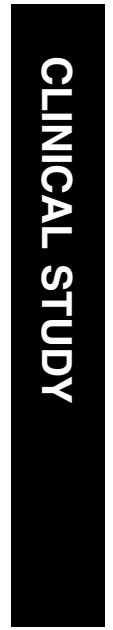

${ }^{1}$ Department of Epidemiology and International Eye Health Institute of Ophthalmology University College London UK

\section{${ }^{2}$ Department of Ophthalmology Chulalongkorn University Hospital \\ Bangkok, Thailand}

${ }^{3}$ Department of Infectious and Tropical Diseases Clinical Research Unit London School of Hygiene and Tropical Medicine London, UK

\section{${ }^{4}$ Vitreo-Retinal Unit St Thomas' Hospital London, UK}

\section{Correspondence:} RRA Bourne International Centre for Eye Health 11-43 Bath Street London EC1V 9EL, UK Tel: +440 2076086907 Fax: +4402072503207 E-mail: rupert_bourne@ hotmail.com

Received: 14 June 2002 Accepted in revised form: 18 December 2002

The authors have no proprietary interest in the RLME chart

\title{
Evaluating a new logMAR chart designed to improve visual acuity assessment in population-based surveys
}

Abstract

Aims This study aimed to evaluate a new chart designed to improve the collection of visual acuity data in population-based surveys. The Reduced logMAR E chart (RLME) employs three letters per line, 'tumbling $E^{\prime}$ optotypes, and conforms to accepted contemporary design principles. Methods The performance of the chart was assessed within a population-based glaucoma survey in Thailand. Performance indices were test-retest variability (TRV) and agreement with acuity data measured using the ETDRS logMAR chart which acted as the 'gold standard'.

Results The $95 \%$ confidence limits for TRV of RLME acuity data were $\pm 0.15 \log$ MAR. This figure is consistent with published data on the TRV of acuities measured using five-letter-perline logMAR charts. The mean difference between RLME and ETDRS acuity data was 0.00 logMAR $(95 \%$ confidence intervals of $\pm 0.05 \log M A R)$ indicating that RLME acuities agreed well with those of the ETDRS chart. The chart and its method of use was readily accepted by the local ancillary staff who required only minimal training before acuity measurement could be delegated to them. Conclusions The study demonstrated that the RLME chart is capable of accurate and repeatable acuity measurements. Certain aspects of the design of the RLME chart may be particularly pertinent to the measurement of vision in population-based surveys. Eye (2003) 17, 754-758. doi:10.1038/ sj.eye. 6700500

Keywords: logMAR; visual acuity; tumbling E; methods comparison study
RRA Bourne', DA Rosser', P Sukudom², B Dineen ${ }^{3}$, DAH Laidlaw', GJ Johnson' and IE Murdoch'

Introduction

Printed panel charts featuring optotypes (letters or other symbols) of varying sizes are a quick and inexpensive method of assessing the spatial resolving capacity of the visual system. As such, they are well suited for use in population-based surveys where visual acuity is often an important outcome measure, and where time and resources may be limited. Many such surveys are conducted on populations in which few subjects are familiar with the Roman alphabet. ${ }^{1-3}$ The problem can be overcome by using 'tumbling' optotypes (identical optotypes presented in one of a discrete number of varying orientations) such as the Tumbling $\mathrm{E}$ and Landolt $\mathrm{C}$, which require only the orientation of the optotype to be elicited rather than the name. This has the advantage of allowing the same chart to be used in numerous populations, enabling a direct comparison of results.

A tumbling E logMAR chart for use in 'field studies' has been described by Taylor. ${ }^{4}$ That chart features five optotypes per line and a range of +0.5 to $-0.5 \log$ MAR. A chart with a greater range of letter sizes would allow a wider range of acuities to be measured for a given distance. Also a reduced number of optotypes per line may allow a balance between ease of test administration and test-retest variability (TRV) which is appropriate for populationbased studies.

This paper describes the evaluation of a new visual acuity chart, the Reduced $\log$ MAR E (or RLME) chart, designed to optimise visual acuity data collection in population-based surveys. The chart utilises those design principles currently advocated for the measurement of visual acuity in clinical 
research, ${ }^{5}$ and employs Tumbling E optotypes. This trial was designed to assess the level of agreement of RLME acuity data with those of the ETDRS chart (which acted as the 'gold standard'), and the TRV of RLME acuity data.

\section{Materials and methods}

The evaluation of the RLME chart was carried out in conjunction with a population-based glaucoma survey in Rom Klao, a suburb of Bangkok, Thailand between December 1999 and February 2000.

Subjects were recruited from those entering the glaucoma survey. A total of 21 consecutive subjects aged over 50 and undergoing assessment as part of the glaucoma survey were requested to undergo additional visual acuity measurements after an explanation of the study. The only additional inclusion criterion for participation in the RLME chart evaluation was familiarity with the Roman alphabet, such that subjects could read both the RLME and ETDRS charts.

The designs of the acuity charts are summarised in Figure 1a and Table 1.

\section{Charts}

The RLME chart employed the design principles of the ETDRS chart ${ }^{4}$ in terms of logarithmic letter size progression, interletter spacing and interline spacing. The RLME chart differed from the ETDRS chart in the use of Tumbling E optotypes, and in having only three optotypes per line. In addition, the chart was modified in an attempt to provide consistency with respect to contour interaction. Contour interaction (also known as crowding) is a well-known phenomenon whereby the legibility of an optotype of a given size depends upon its proximity to surrounding optotypes. ${ }^{6}$ The chart was therefore surrounded by a continuous 'crowding bar' to compensate for the fact that the number of optotypes crowded from both sides has reduced from three, in the case of the ETDRS chart, to one in the RLME chart. This bar was positioned 2.5 stroke widths (half an optotype) from the edge of the optotypes, as the difficulty of letters crowded in this way has been shown to equate well to that of letters in a linear arrangement ${ }^{7}$. A chart featuring letters from the Sloan set, but otherwise identical to the RLME chart, has been evaluated in a previous study. ${ }^{8}$ Two versions of the RLME chart were used, differing only in the orientations of the optotypes. ETDRS chart 1 (Lighthouse International) was used for purposes of comparison of visual acuity results with the RLME chart.

\section{Testing protocol}

The visual acuity testing process was first explained to the subject. Acuity measurements were conducted on
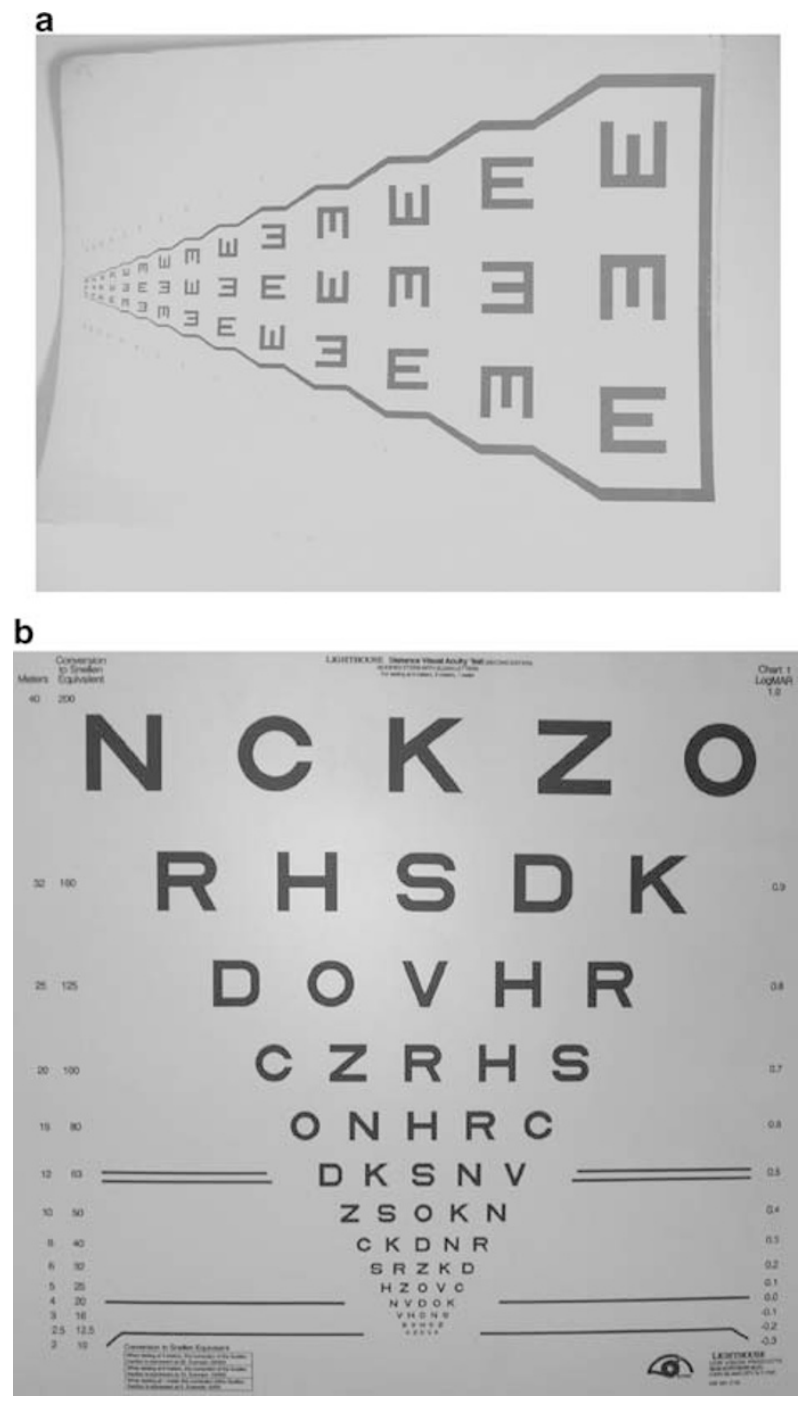

Figure 1 The RLME chart (a) and the ETDRS chart (b).

one eye of each subject by a single examiner under consistent indoor lighting conditions. To limit psychological sources of variability, a forced choice paradigm was employed such that subjects were required to respond whether or not they felt they were capable of a correct response. Both charts were displayed in a consistent indoor environment under diffuse artificial light. Measurements were taken on the right eye of each subject using an RLME chart followed by the ETDRS chart followed by the alternate version of the RLME chart. The chart order was reversed for each subject to avoid any bias because of learning effects. Subjects responded to each optotype either by indicating the direction of the letter $\mathrm{E}$ using their hands or by matching the orientation of the optotype on the chart with that on a card held by the subject. The method of indicating the orientation of the ' $\mathrm{E}$ ' optotypes was 
Table 1 Summary of the design of each chart

\begin{tabular}{lccccc}
\hline Chart & $\begin{array}{c}\text { No. of letters } \\
\text { per line }\end{array}$ & $\begin{array}{c}\text { Line interval } \\
\text { in } \log M A R\end{array}$ & $\begin{array}{c}\text { Single-letter value } \\
\text { in logMAR }\end{array}$ & Total no. of letters & Acuity range at $4 \mathrm{~m}$ \\
\hline ETDRS & 5 & 0.1 & 0.02 & 70 & +1.00 to -0.30 \\
RLME & 3 & 0.1 & 0.0333 & 42 & +1.00 to -0.30 \\
\hline
\end{tabular}

determined according to which seemed to be easier or more comprehensible for each subject during the test. Subjects were tested monocularly wearing their habitual distance spectacle correction if they possessed one, or unaided if they did not. The measurement method was explained by showing the subject several sample E optotypes, which are then matched for orientation to one of the four possible E orientations displayed on a card held by the patient. Following the demonstration, responses were elicited using either matching or hand gestures depending on subject preference. Charts were viewed from a distance of $4 \mathrm{~m}$ unless the subject made any incorrect responses on the top line of a given chartindicating an acuity worse than $+1.00 \log$ MAR (6/60 Snellen). In this event, the subject was moved to $1 \mathrm{~m}$ and the remainder of the testing process was performed at this distance. The VA testing continued until the subject incorrectly responded to an entire line of optotypes or, in the case of any correct responses on the bottom line, all the optotypes on the chart having been attempted. The number of correct subject responses was counted by a single examiner using a hand-held tally counter and recorded on a specifically designed data proforma, allowing the acuity scores to be calculated subsequently.

\section{Scoring}

An interpolated logMAR acuity score ('single-letter' score) was produced for all acuity measurements using the formula

$$
+1.10-T_{\mathrm{C}} L_{\mathrm{V}} \log \mathrm{MAR}
$$

where $T_{\mathrm{C}}$ is the total number of correct responses and $L_{\mathrm{V}}$ is the $\log$ MAR value of each letter on the chart. The individual letter values are produced by dividing the $\log$ MAR interval between a given line and the line below by the number of letters on that line. Hence, $L_{\mathrm{V}}$ for the ETDRS chart will be $0.02 \log$ MAR, whereas for the RLME chart it will be $0.0333 \log$ MAR.

\section{Method of quantitative analysis}

The methods of Bland and Altman ${ }^{9}$ were used to determine both the extent to which measurements taken using the RLME chart agreed with the 'gold standard' ETDRS chart, and the TRV of RLME acuity data. The level of agreement is indicated by the mean difference between paired measurements taken using the two charts, and the 95\% confidence interval for this mean. TRV is indicated by the $95 \%$ confidence limits for the differences of paired measurements taken using the RLME chart only.

\section{Results}

A total of 21 subjects were recruited with a median age of 61 (ranging from 52 to 69). The acuities ranged from 0.00 to $+0.72 \log$ MAR (6/ 6 to $6 / 31$ Snellen equivalent) with a median of $+0.26 \log$ MAR (6/11 Snellen).

The mean difference between paired measurements taken on the RLME 1 chart and the ETDRS chart was $-0.006 \log$ MAR $(95 \%$ confidence interval \pm 0.04 $\log$ MAR). The differences between paired measurements were plotted against their mean to assess any effect of underlying acuity upon the extent of agreement between RLME and ETDRS data (see Figure 2). This suggests that the RLME chart produces valid acuity data when compared with the ETDRS chart.

The difference between test and retest visual acuity, using the RLM chart only, is illustrated in Figure 3. The mean difference (or 'learning effect') was an improvement of $0.025 \log$ MAR $(95 \%$ confidence interval $\pm 0.033 \log \mathrm{MAR})$

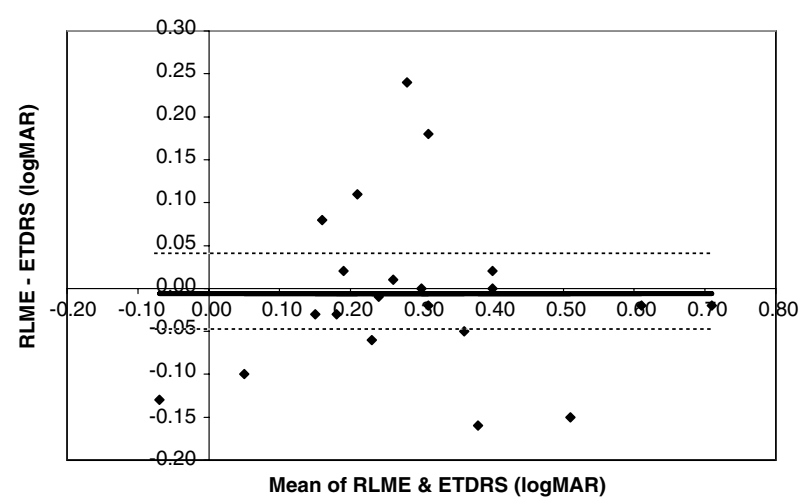

Figure 2 Effect of underlying acuity upon agreement of between RLME acuities with ETDRS acuities. The bold horizontal line gives the mean difference $(-0.006 \operatorname{logMAR})$ between visual acuities measured by each chart, and the broken horizontal lines give the upper $(+0.036 \log \mathrm{MAR})$ and lower $(-0.048) 95 \%$ confidence limits. 


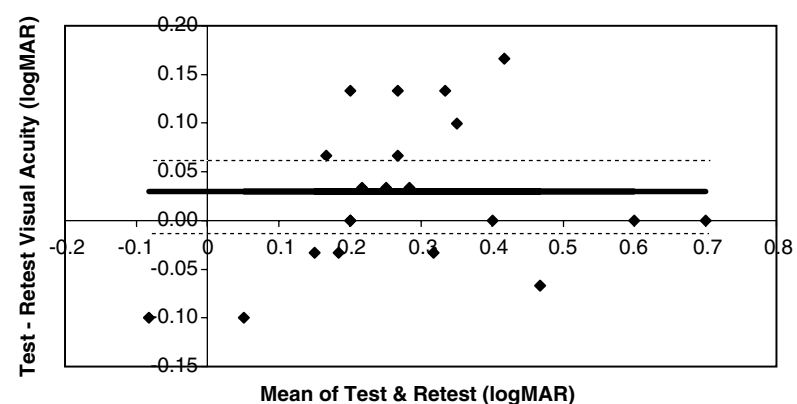

Figure 3 Effect of underlying visual acuity on test and retest visual acuity using the RLME chart only. The dashed lines indicate the mean difference and bold lines the upper and lower 95\% confidence limits. The bold horizontal line gives the mean difference between test and retest $(+0.025 \log M A R)$, and the broken horizontal lines the upper $(+0.058 \log$ MAR $)$ and lower $(-0.007) 95 \%$ confidence limits.

The 95\% confidence limits for TRV were \pm 0.15 $\log$ MAR indicating that $95 \%$ of repeated RLME acuity measurements on eyes with stable vision will fall within $\pm 0.17 \log \mathrm{MAR}$ of one another (this figure is achieved by rounding up to the next scale increment).

\section{Discussion}

Recent years have seen a general consensus develop as to the preferred design principles of visual acuity charts for use in clinical research. ${ }^{5}$ It would seem advantageous for these principles to be applied uniformly to all situations in which acuity is measured, providing there are no inherent disadvantages in doing so.

The results of the present study suggest that a logMAR Tumbling E chart such as that used here can produce acuity data that agree well with those produced by the ETDRS logMAR chart, which acted as gold standard. The absence of systematic bias resulting from this analysis was noteworthy as the small number of possible orientations using a Tumbling E chart might have been expected to produce better acuities, as a correct guess is more likely. The level of TRV achieved with the RLME chart (95\% confidence limits of $\pm 0.15 \log$ MAR) is consistent with previous studies, which measured the TRV of five-letter-per-row logMAR charts which range from \pm 0.09 to $\pm 0.20 .^{10-15}$
The number of optotypes per line on the E chart was limited to three to improve the ease of administration. Although this study did not assess test time, a previous study ${ }^{8}$ found that a letter chart featuring an identical design to the RLME chart allowed measurements to be taken in half the time of the fiveletter-per-row ETDRS chart. We would anticipate the time saving with the E chart to be of a similar magnitude, which would be very desirable for population-based surveys. Unpublished data on a separate group of 28 subjects gave a median test duration using the RLME chart of $86 \mathrm{~s}$.

The advantages of applying standardised design principles to visual acuity charts have been well described $^{16,17}$ and are summarised in Table 2.

There may, however, be particular advantages in employing such an approach in the case of populationbased surveys:

(a) The geometric nature of optotype size progression allows the measurement of a wide range of acuities by altering the distance from which the subject views the chart.

(b) In being regarded as interval data, the acuity data produced by these charts are amenable to parametric statistical analyses, which are not applicable to Snellen acuity data.

(c) In addition to the increased sensitivity to change allowed by single letter scoring, ${ }^{12}$ single letter scoring carried out with a teller counter can offer other advantages if acuity cutoffs are employed in the study. For example, for WHO Category 1 visual impairment, ${ }^{18}$ the subject must have an acuity less than $6 / 18$, but equal or better than $6 / 60$ Snellen $(0.48 \leq 1.0 \log M A R)$. This equates to the ability to name at least three optotypes on the RLME chart correctly at $4 \mathrm{~m}$, but fewer than 19. Using a Snellen chart, a subject achieving, for example, $6 / 24$ but having made numerous mistakes on preceding lines cannot be differentiated from one who has made no mistakes up to that point.

(d) The logMAR acuity data can be readily converted into Snellen acuity notation for those unfamiliar with $\log$ MAR notation.

Table 2 The advantages of logMAR charts

\begin{tabular}{ll}
\hline Design feature & Advantage \\
\hline $\begin{array}{l}\text { 1. Equal number of optotypes per line } \\
\text { 2. Equal logarithmic interval between lines }\end{array}$ & Allow the use of single-letter scoring which reduces test-retest variability \\
$\begin{array}{l}\text { 3. Equal average legibility for each line } \\
\text { 4. Consistent spacing between letters and lines }\end{array}$ & Ensure that letter size is the sole determinant of difficulty on a given line \\
5. Geometric progression of letter sizes & Allow testing distance to be varied \\
\hline
\end{tabular}


(e) The approach of counting correct responses with a teller counter is relatively easy to explain thereby simplifying delegation of the measurement to ancillary staff.

\section{Acknowledgements}

We thank Mr Prin Rojanapongpun (Department of Ophthalmology), and Professor Sutthichai Jitapunkul (Department of Geriatric Medicine) of Chulalongkorn University Hospital who authorised and invited ICEH to collaborate with the Rom Klao Glaucoma Survey. Dr Wongwat Luiwlak, the director of the Community Health Centre in Rom Klao, gave logistic support, and Mr Pak Sang Lee (Institute of Ophthalmology, London ICEH) provided technical aid. Ms Benjamas Prapamont, Ms Nattinee Riandara, Ms Siriwan Chatapatama, Ms Wanna Kittiyapison, and Miss Ranee Taveekiteekul recruited subjects and assisted with the study.

Grant support: British Council for Prevention of Blindness; The Glaucoma Research Fund (Thailand); Special Trustees, Moorfields Eye Hospital, London, UK.

\section{References}

1 Mansour AM, Kassak K, Chaya M, Hourani T, Sibai A, Alameddine MN. National survey of blindness and low vision in Lebanon. Br J Ophthalmol 1997; 81(10): 905-906.

2 Pokharel GP, Regmi G, Shrestha SK, Negrel AD, Ellwein LB. Prevalence of blindness and cataract surgery in Nepal. $\mathrm{Br} J$ Ophthalmol 1998; 82(6): 600-605.

3 Zerihun N, Mabey D. Blindness and low vision in Jimma Zone, Ethopia: results of a population-based survey. Ophthalmic Epidemiol 1997; 4(1): 19-26.

4 Taylor HR. Applying new design principles to the construction of an Illiterate E chart. Am J Optom Physiol Opt 1977; 55: 348-351.
5 Ferris FL, Bailey I. Standardizing the measurement of visual acuity for clinical research studies: Guidelines from the Eye Care Technology Forum. Ophthalmology 1996; 103(1): 181-182.

6 Flom MC, Weymouth FW, Kahneman D. Visual resolution and contour interaction. Opt Soc Am 1963; 53(9): 1026-1032.

7 Stager DR, Everett ME, Birch EE. Comparison of linear and optotype acuity in amblyopia. Am Orthop J 1990; 40: 51-56.

8 Rosser DA, Laidlaw DAH, Murdoch IE. The development of a 'reduced $\log M A R^{\prime}$ visual acuity chart for use in routine clinical practice. Br J Ophthalmol 2001; 85: 432-436.

9 Bland J, Altman D. Statistical methods for assessing agreement between two methods of clinical measurement. Lancet 1986; i: 307-310.

10 Siderov J, Tiu AL. Variability of measurements of visual acuity in a large eye clinic. Acta Ophthalmol Scand 1999; 77(6): 673-676.

11 Elliott DB, Sheridan M. The use of accurate visual acuity measurements in clinical anti-cataract formulation trials. Ophthalmic Physiol Opt 1988; 8(4): 397-401.

12 Bailey I, Bulimore M, Thomas W, Taylor H. Clinical grading and the effects of scaling. Invest Ophthalmol Vis Sci 1991; 32: 422-432.

13 Arditi A, Cagenello R. On the statistical reliability of letterchart visual acuity measurements. Invest Ophthalmol Vis Sci 1993; 34(1): 120-129.

14 Lovie Kitchin JE. Validity and reliability of visual acuity measurements. Ophthalmic Physiol Opt 1988; 8(4): 363-370.

15 Reeves B, Wood J, Hill A. Vistech VCTS 6500 charts, withinand between-session reliability. Optom Vis Sci 1991; 68(9): 728-737.

16 Bailey I, Lovie J. New design principles for visual acuity letter charts. Am J Optom Physiol Opt 1976; 53: 740-745.

17 Ferris FL, Kassoff A, Bresnick GH, Bailey I. New visual acuity charts for clinical research. Am J Ophthalmol 1982; 94: 92-96.

18 World Health Organization (WHO). Methods of Assessment of Avoidable Blindness. WHO Offset Publication No. 54. WHO: Geneva, 1980. 